\title{
Spreading Corrosion-Model of Self-Potential Methods, Case Study of Buried Metal Laboratory Scale
}

\author{
Lutfian Rusdi Daryono ${ }^{1, *}$, Rentyas Hellis Rahmadani Surya ${ }^{2}$, Imam Suryanto $^{2}$ \\ ${ }^{1}$ Lab. Biotechnology for Resources, Division of Sustainable Resources, Graduate School of Engineering \\ Hokkaido University, Japan \\ ${ }^{2}$ Geophysics Sub-Department, Faculty of Math and Science \\ University Gadjah Mada, Indonesia \\ *Corresponding author's email: luthfian.daryono [AT] gmail.com
}

\begin{abstract}
Self-potential is a geophysical method as an inexpensive, practical, and simply used measurements. Measuring with this as simply used, make it's become sensitive to noise and causing difficulties with interpretation. As an environment geophysical method, self-potential would give a good interpretational model. One other way to getting a good model to reduced the noise data. The noise reduction developed by making experiment box model as the only exploration field. Experiment box model is formed by composing paving blocks at the edge of the box. The purpose of the experiment is to analyze the target, which is a massive iron cylinder, as a physical and chemical reaction. The iron placed in the sandbox which has two conditions, half-down wet and half-up dry. The porous pot made from mini straw which is appropriated with the sandbox dimension and target experiment. The experiment took five weeks as periodically measurement.
\end{abstract}

The results were potential graphics and two-dimension iso-potential maps. Existing of iron-related to large-amplitude potential anomalies around the target. Thus, corrosion-related to compared periodical-maps of anomaly contour area. The difference of anomaly's pattern in the last two maps might cause the internal changed of the box model. This experiment indicated that self-potential signals can be used for monitor corrosion processes.

Keywords- Self-potential, anomalies, corrosion processes, laboratory experiments.

\section{INTRODUCTION}

Subsurface mapping for geophysics major had a lot of application purposes, for mining industries, contamination mapping area, disaster mitigation purpose, or other purposes. Impact of this evolution method for delineation subsurface structure or imagine more specific, accurate, and clearly imagined. Self-potential (SP) is one of the geophysical methods based on electricity principal that has been used since the year 1830 by Robert Fox for metal exploration (Revil and Jarandi, 2013). The self-potential method is the oldest geophysical methods, conducted passively, cheap, fast, and simple. Therefore, these measures tend to be sensitive to noise and cause trouble when interpreting the results. Because of the simplicity and cheapest method, SP could have detected the potential water distribution area or metal minerals beneath the ground. The environment geophysics topics, SP is one of the main methods that can generate a good model (Rotht, et. al., 2002).

The changes in the parameter of potential value in the field during measurement may be known as the systematic investigation on the survey area, permanently, for several days. According to Ernstson and Scherer (1986), the parameter was composed by the root of vegetation activity, soil temperature, lithology and movement of groundwater surface. Lithology, in this case, related to the body of the minerals beneath the surface. The potential value is given of negative variations up to a positive anomaly. The value of self-potential anomalies is associated with sulfide minerals occurred to the electric current formed by the reaction of oxidation and reduction among most mineral body wet and dry others (Sato and Mooney, 1960). Results of the geophysical data measurements for imagine geo-structural sub-surface based on cross-section models. To get the best subsurface models, it involves the reduction of the noise in the data. Reducing noise in self-potential methods, will analogize the artificial pool as measurement medium, with the target as metal cylinder beneath the ground medium. The purpose of this research is to identify locations target (precision) and corrosion process on the target surveys within assumed graphics and iso-potential 2D-map.

\subsection{Electrochemical Cells}

Corrosion of metal occurs during electrochemical reactions. These reactions are described as laboratory experiment models of electrochemical cells (Figure 1). In the figure below, two plates of different metals, which are zinc and copper, 
and submerged in salt and liquid solutions. These differences in metals, trigger the chemical reactions, zinc metal will be oxidized. Electrons come from oxidation, going to positive current as copper plate metals bypassing the wire.

Copperplate is subjected to reduction process. Oxidation plate, zinc, is called anodes whereas reduction plates, copper, is called cathode. An electron that passes through the wires generated electrical current based on different potential between the tip of the wire.

Corrosion process in this model, because half came from the position of a metal target that is placed in the saturated medium and other half is unsaturated (dry) (Figure 2). A chemical reaction occurred as oxidation beneath the metal plate and electron from that process running up the surrounded top of the metal plate. These electrons will be used with oxygen in the reduction of water into hydroxide. Meanwhile, oxygen at the top of the metal plate, was diffusing between pores of rock flowing beneath the metals and produced oxide rust.

\section{MATERIAL AND METHODS}

This research is using mapping and monitoring function methods. Mapping measurement using fixed-based configuration, whereas poles that is connected with reference point is the negative pole. This measurement is conducted for 29 days and it is described as 6 samples data, shown in table 1 below.

\subsection{Porous Pot}

Porous pot made from plastics straw, figure 3, within $\mathrm{Cu} / \mathrm{CuSO} 4$ liquid insides. Measurement instrument potential meter using JLM Thermospro, with 24-bit analog-to-digital converters and 4 poles channel.

\subsection{Measurement Pool Models}

This research is conducted in the miniature pool with size $120 \mathrm{~cm} \times 80 \mathrm{~cm} \times 46 \mathrm{~cm}$, and the target area is $103 \mathrm{~cm} \times 62$ $\mathrm{cm} \times 20 \mathrm{~cm}$. Shaving sand as a medium inside the miniature pool for testing electrodes. Research target metal iron cylinders with size $15 \mathrm{~cm}$ tall and $4 \mathrm{~cm}$ diameters. Target will be buried in medium, vertical, between two layers of sand medium. $6 \mathrm{~cm}$ in the saturated medium and $9 \mathrm{~cm}$ in the unsaturated (dry) medium. Reference point position was left the side top of the measurement pool (Figure 4).

\section{RESULTS}

\subsection{Graphic Analysis}

Targets position can be identified by potential amplitude from the graphics below. Graphic T-1 noted targets position in $40 \mathrm{x}$-axes or the 9th column. The zone affected from the anomalous zones is illustrated with colorful areas, while the un-affected areas is illustrated as point and lines. Those potentials amplitude had a negative value under the anomalous zones. The occurrences process on the target indicated as corrosion process, known as a comparison between spacious of the anomalous area. Based on the graphic T-1 (Fig.5) and graphic T-9 (Fig.6), noted as colorful areas more widely mean the anomalous zone was wider too.

Meanwhile, were conducting monitoring method measurement as related to the time function. The observation measuring for 14,5 hours, within 4-point position observation. Which is row 1 , row 3 , row 6 , and row 6 ? Based on that points, sighted as the same pattern in the monitoring graphics row 1, row 6, and row 9 (Figure 7). Potentials amplitude maximum between 13.12 until 15.00, Indonesian time zone. This situation occurred from the humidity factor differences in the surface sand medium. Sunlight intensity became one of the factors that is impacted in the self-potential measurement for this observation in the surface of the sand medium in the measurement pool. Graphic Monitoring Row 3 has a different pattern because of impacted targets located around that point.

High potential values, based on that monitoring points $<1 \mathrm{mV}$, inversely the mapping data is around the anomalous zones close to $\sim 70 \mathrm{mV}$. Graphic monitoring is only $10 \%$ if compared with mapping data of self-potential observation, afterward the assumed value does not need to be diurnal corrected.

\subsection{Iso-potential Maps Analysis}

Iso-potential maps delineated as contouring potential value, which is a potential value from measurement data that will be classified by one of contoured line. Color scale in the potential maps had a $2 \mathrm{mV}$ range scales, all the data. Isopotential maps could be an analysis based on (a) the color of the contour point locations target; (b) anomalous zone; (c) the color of contour that did not impact on the anomaly. Those three can be categorized into quantitative analysis based on contour maps. Target locations could be identified based on the color contrast in the anomalous zone, comparing with target iso-potential maps will have a small differences potential value in the figure 8.b. No targets (metal buried) shows that there is no contrasting color of the contour, figure 8.a., shows the target locations had a color contrast.

Corrosion process that happened on the target can be known from the compared data potential maps (Figure 9). Differences in color distributions contour pattern represent differences in the anomalous zone areas. That means, rust oxide impacted areas influenced the time difference. The differences pattern that assuming as rust oxide distributions or redox reaction had unique characteristics one and another, the distribution of corrosion process. Non-linear anomalous covered areas could be impacted based on several factors. First assumption is iron oxide consist of redox reactions which 
does not have the same pattern like at the beginning. The second assumption is sand humidity, water saturated inside sand particles were not same between T-9 and T-14, resulting in water as oxidizing agent and/ or electron carrier no longer impacting as before, not homogenous pores sand medium caused permeability of water saturation were not the same as before. The intensity of sunlight perhaps changed the temperature of the medium.

The third assumption is the existence of acidic medium. Before the targeted metal was buried beneath the medium, this metal was cleaned with hydrochloric acid (HCL) surrounding the cylinder metal target. Hydrochloric acid content might trigger the corrosion process that caused the reaction accelerating in the T-4 and T-14.

Corrosion process in the metal may occur from water contact in the bottom of the metal plate and oxygen contact on the top surface. Redox reactions, shown in the $5.1 \sim 5.3$ equations, that metals plate had iron oxide:

$$
\mathrm{Fe} \rightarrow \mathrm{Fe}^{2+}+2 e^{-}
$$

Afterward oxidation reactions keep going inside iron ion (II) and produce iron ion (III):

$$
\mathrm{Fe}^{2+} \rightarrow \mathrm{Fe}^{3+}+e^{-}
$$

Electrons from oxidation reactions result streamed electronically to the surrounding metals body, as a cathode. That means, oxygen and water diffusion as hydroxide ion:

$$
\mathrm{O}_{2(\mathrm{~g})}+2 \mathrm{H}_{2} \mathrm{O}+4 e^{-} \rightarrow 4 \mathrm{OH}^{-}
$$

the electron content in the top of metals led negative value anomalous in the target areas. Rust oxide that occurs, $\mathrm{Fe} 2 \mathrm{O} 3 . \mathrm{xH} 2 \mathrm{O}$, from the reaction between water, oxygen, and iron ion. Oxygen that diffused inside water particle were reacted and forming rusty in the bottom of metals plate (Figure 10).

\section{DISCUSSION}

Data correlation between anomalous zone is based on iso-potential map T-1, until T-9 represents the iron oxide or redox extended surrounding the metal target. However, comparison between T-14 and T-19, shows it should be a widely linear same pattern as the other. The difference in the pattern of the anomalous zone was also explained in the research Rittgers (2013). Non-linear coverage pattern of these anomalies can be caused by several factors. The first possibility, the content of iron oxide or speed of a redox that occurs is no longer the same pattern. The second possibility i.e. moisture of sand. Distribution of water in the sand is no longer the same time T-9 and T-14, therefore the water acted as a carrier agent for oxides or electrons and no longer play a role as the beginning. A homogenous pore medium sand causing the water distribution is no longer the same, or another possibility, deduced was the difference in the temperature encouraged the process of evaporation. A third possibility also was the presence of acid. Before the iron body was placed in its position, the iron surface was cleaned of rust and another with a solution of hydrochloric acid (HCL). Acidic solutions could accelerate the corrosion process, which was evident as shown in the difference in the speed of the reaction between the T-9 and T-14.

A linear continuation pattern happened to the data afterward. Uncertainty based on the possibilities that have been described, the condition of the measurement model was no longer ideal, which led to the process of redox reactions and their products were no longer scattered according to the linear pattern. The study was based on research conducted by Castermant (2008) and Rittgers (2013). The key to differences between these research that has been done with the study of reference namely physical form and the measurement medium. Although reconstruction has a physical form of medium of different measurements, the potential contours patterns imaged zone anomalies have similar results.

The results are obtained, potential negative anomalies of the area surrounded the measurement target. Application of this research could be used for prospective and corrosive mechanism of the iron body beneath the surface, especially for environment geophysics and geophysics engineering major. Nyquist and Corry (2002) for the example, they combined the method in the exploration of the body of pollutants in an area of the mine. They then modified the observation of corrosion of pipe well on a geothermal area, as performed by Campbell (1977).

\section{CONCLUSIONS}

The existence of the target and corrosion process in the metals representing negative anomalous potential respond, based on contrasts potential from graphics and contour maps. And the corrosion process in the metals occurred from the contact between water and oxygen, proven with rust sediment beneath the metals bottom. In the next research to reduce bad effect could be from iron metals as a target which could have been contaminated from pollutants. Like the visible aquarium pool test measurement; homogeneous sand medium, included the humidity, grain size, and other. 


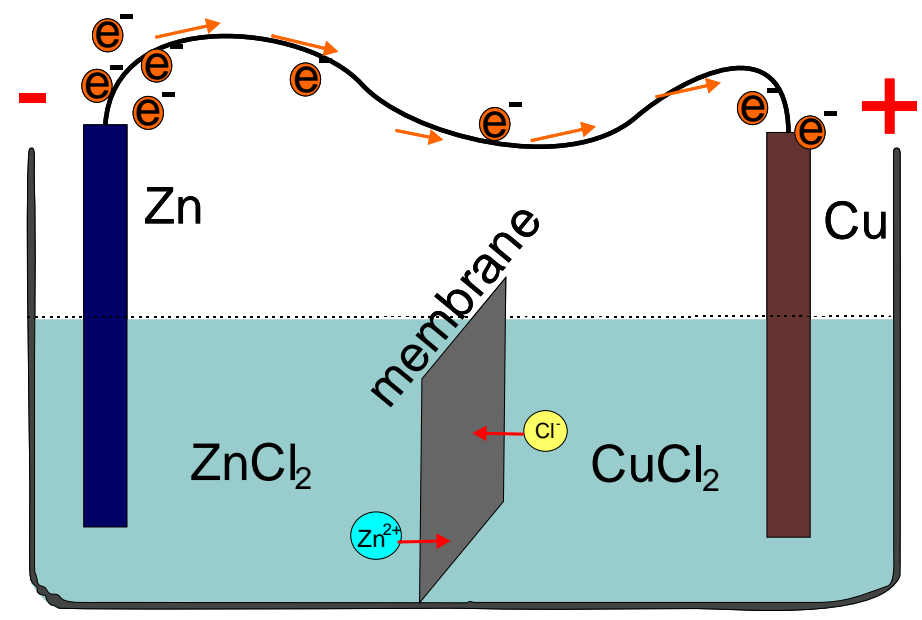

Figure 1: Model of Electrochemical Cells, Reduction Process in The Zinc Plate and Oxidation Process in The Copper Plate (Modified by Lower, 2004).

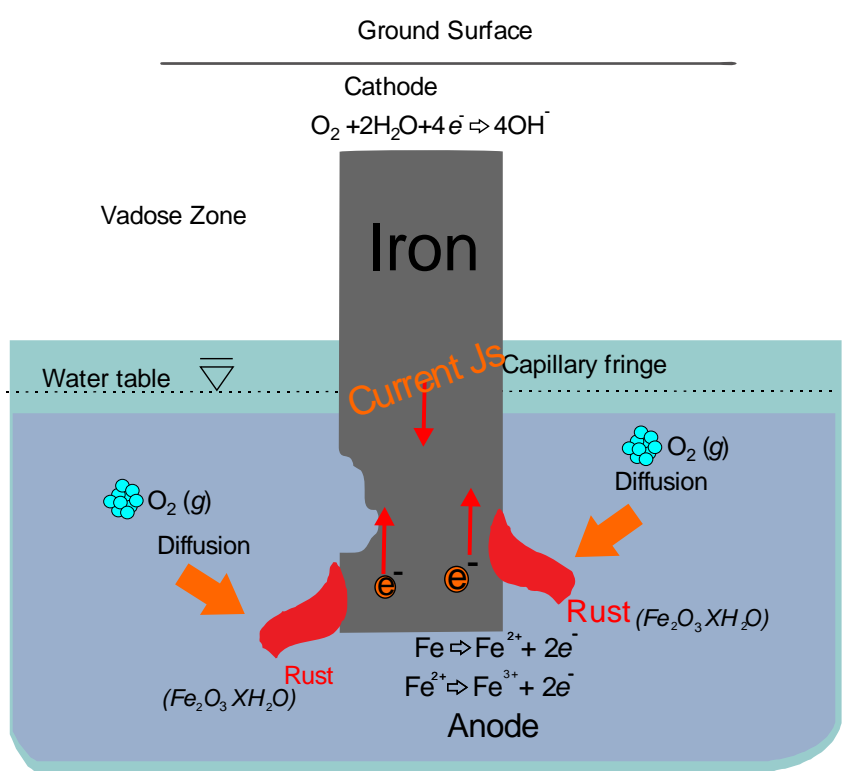

Figure 1. Model Galvani Half-Cells, oxidation process occurs beneath the metal plate. Electrons from oxidation process used for reduction process in the bottom of the metal plate, $\mathrm{O}_{2}$ reacted with metal and water to form oxide rust (Modified by Rittgers, 2013). 

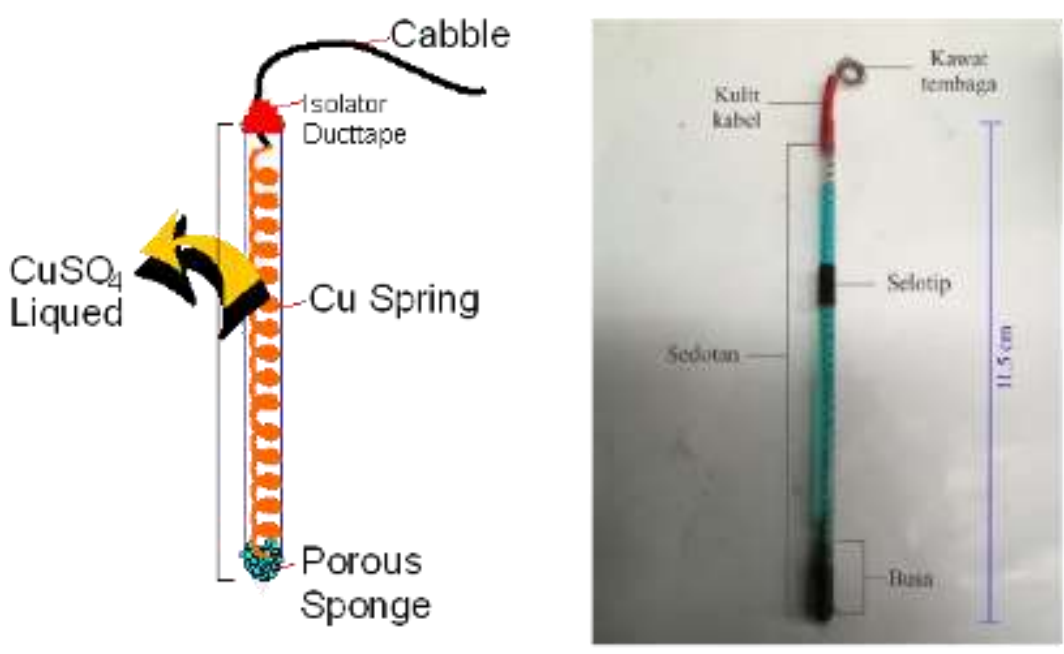

Figure 2. Plastic Straw Porous Pot Electrodes.
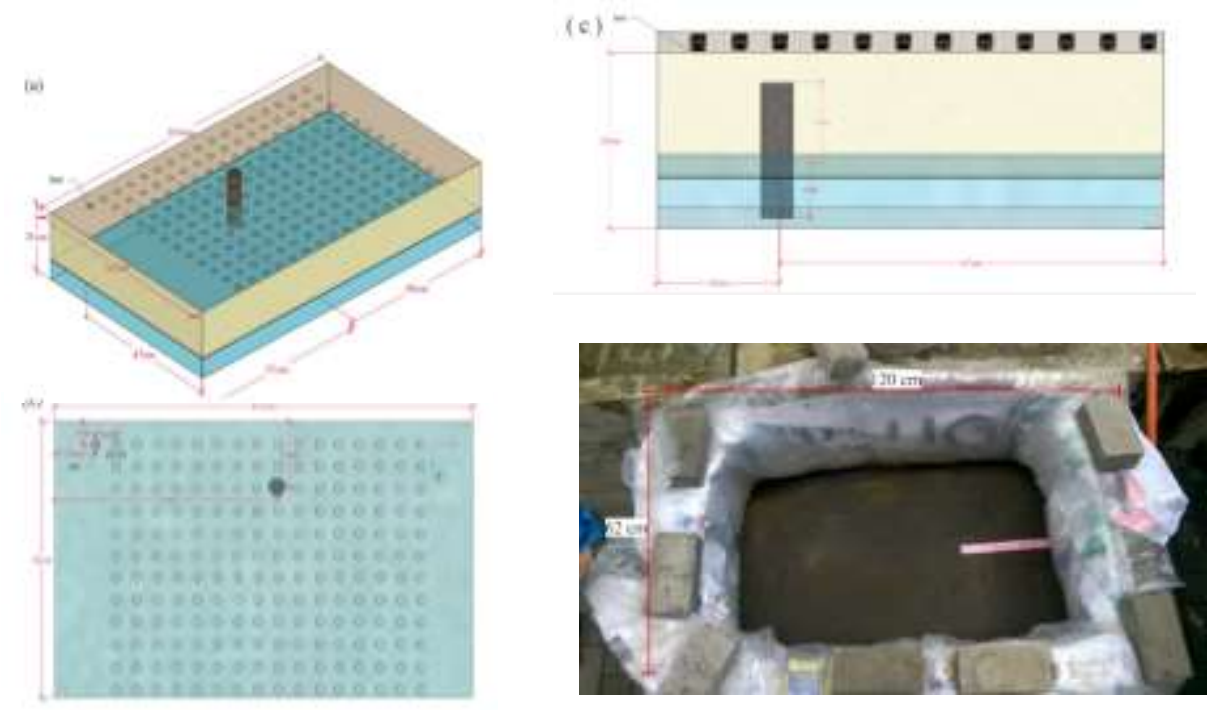

Figure 4. Measurement Pool Models (a) 3D Imagine, (b) Top, (c) Left. Targets positions in the $3^{\text {rd }}$ row and $9^{\text {th }}$ column, whereas vertical placement (half saturated and half un-saturated medium buried).

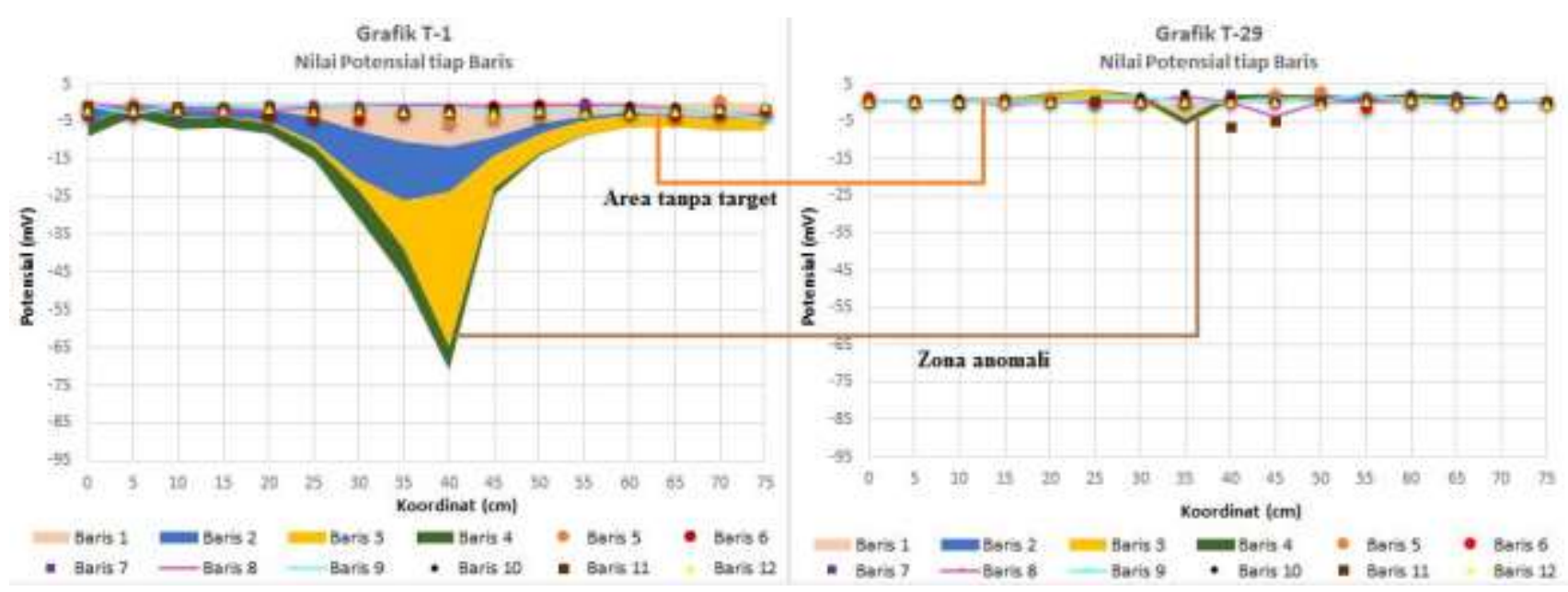

Figure 5. Graphic T-1, potentials amplitude with negative value affected. 


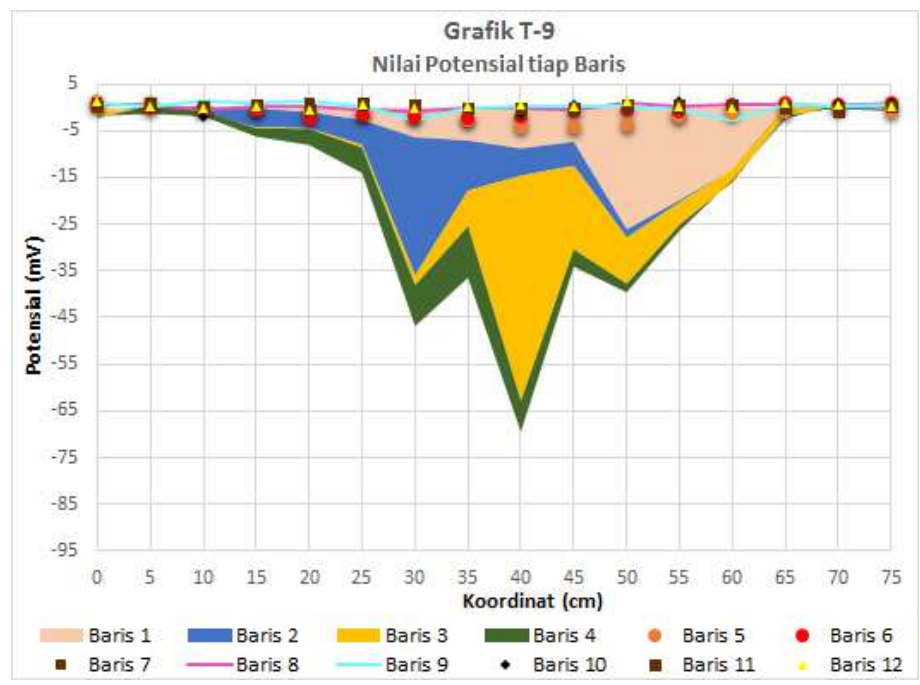

Figure 6. Graphic T-9, spacious anomalous zone represented with colorful Area Pattern with Time Function (wider than the beginning).

(a)

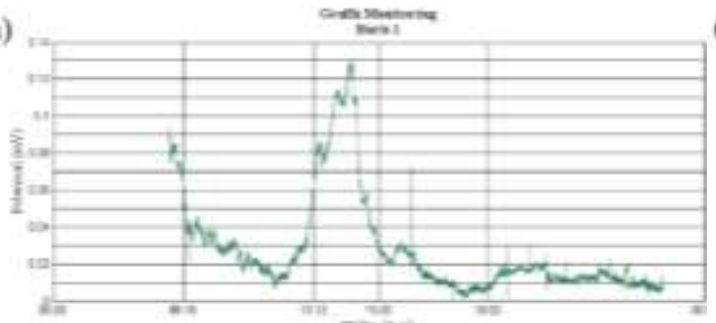

(b)

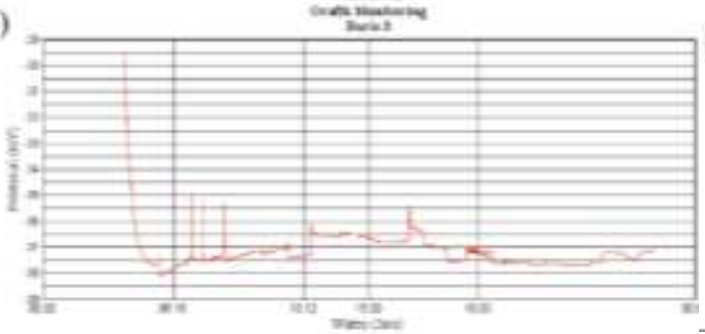

(c)

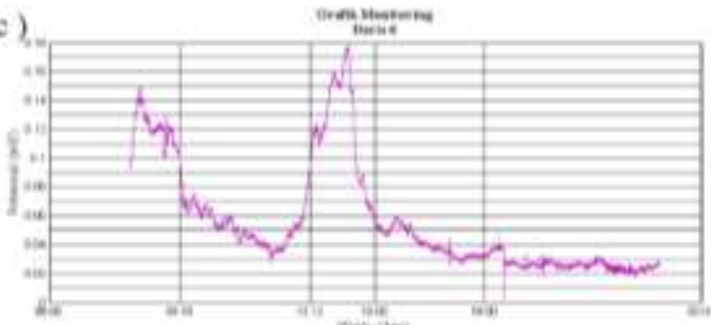

(d)

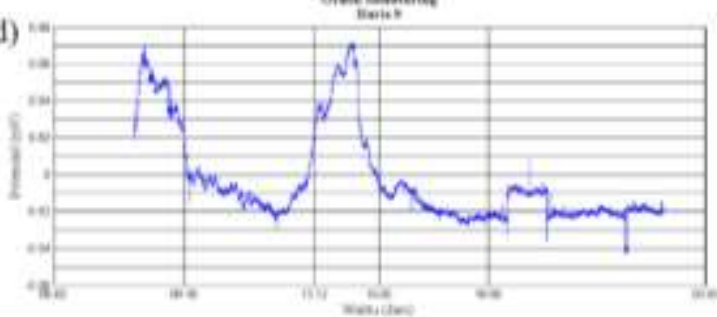

Figure 7. Graphic monitoring, (a) columnar T-1, (b) columnar T-3, (c) columnar T-6, (d) columnar T-9.

(a)

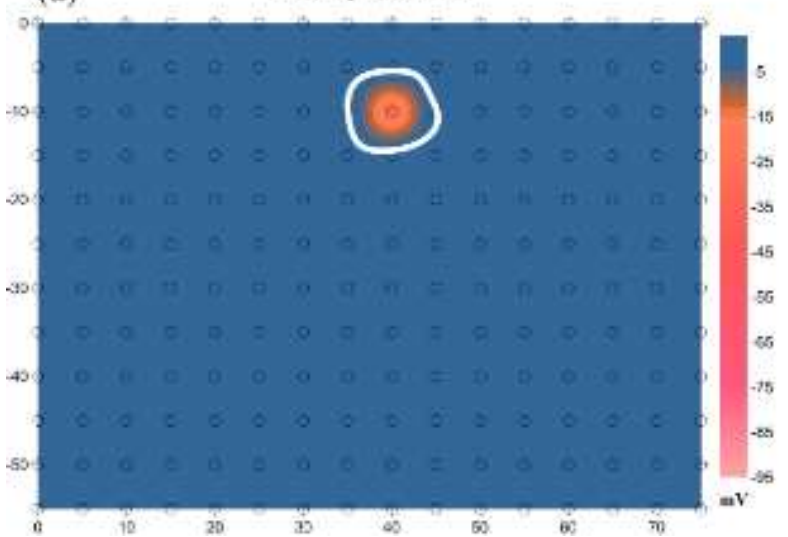

(b)

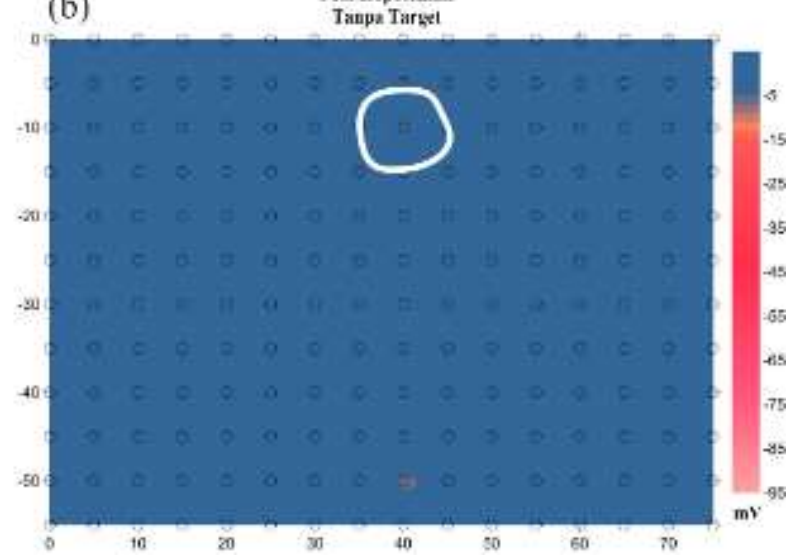

Figure 8. Comparison iso-potential maps, (a) Isopotential T-19 red contrast color represent the target (b) Isopotential map T-29 with no target. 
(a)

Peta lsopotensial T-2

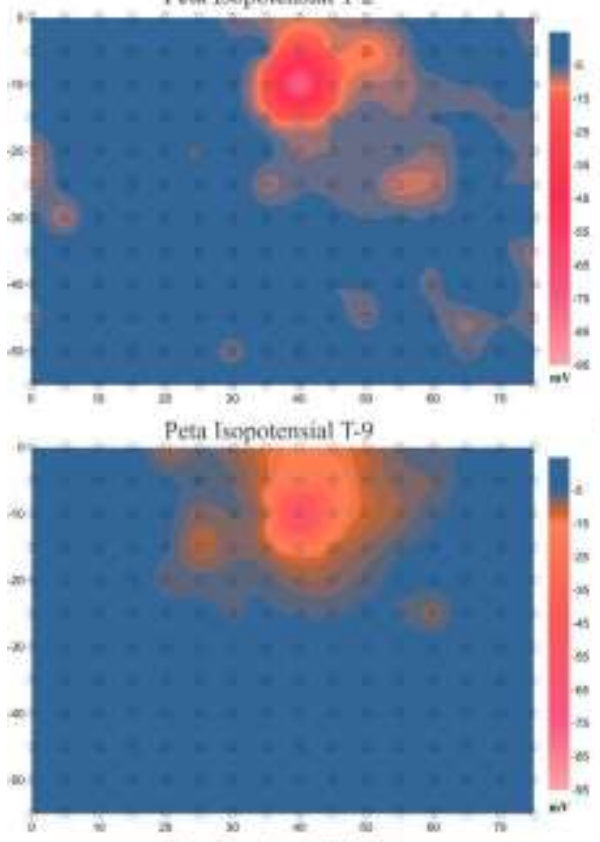

Peta Esypotensial T-14

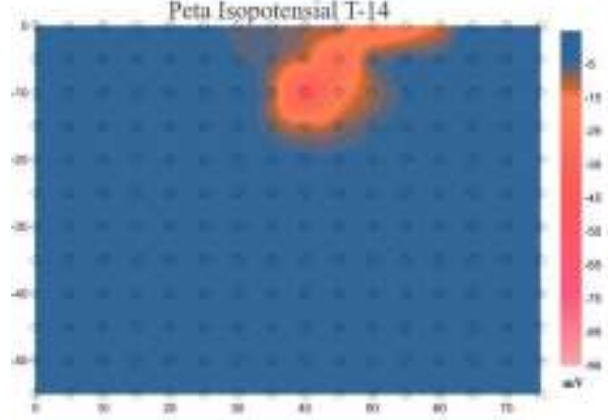

(b)

T4

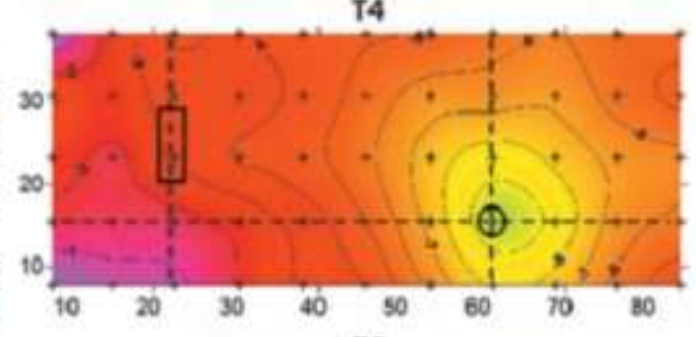

TS

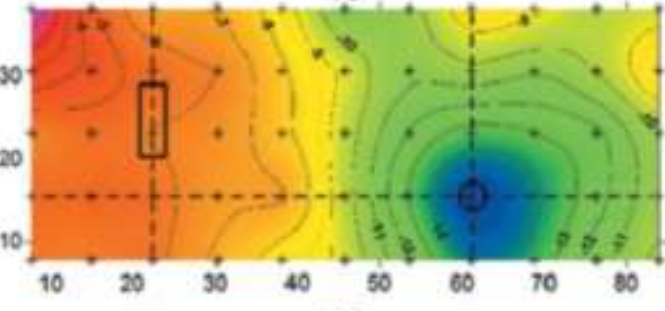

T6

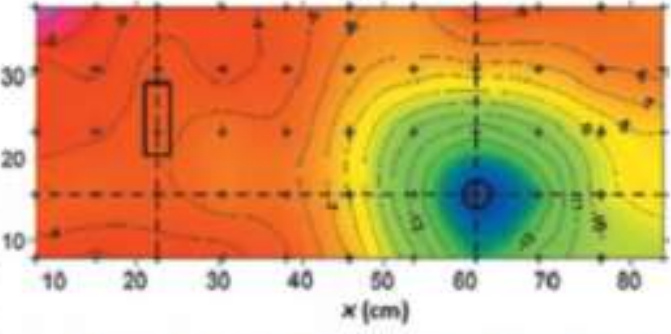

$\operatorname{sp}(\mathrm{mV})$

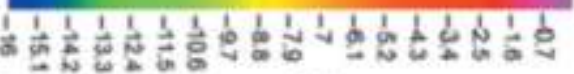

'Location of verical i 20 cross sections Location of

$\prod \begin{gathered}\text { Location of horizontal } \\ \text { buried metalic pipe }\end{gathered}$

O Location of verical

Figure 9. Comparison of iso-potential maps. The non-continuation of the wide anomalous zone pattern is shown in (a) iso-potential measurement maps and in (b) iso-potential maps from Rittgers (2013)

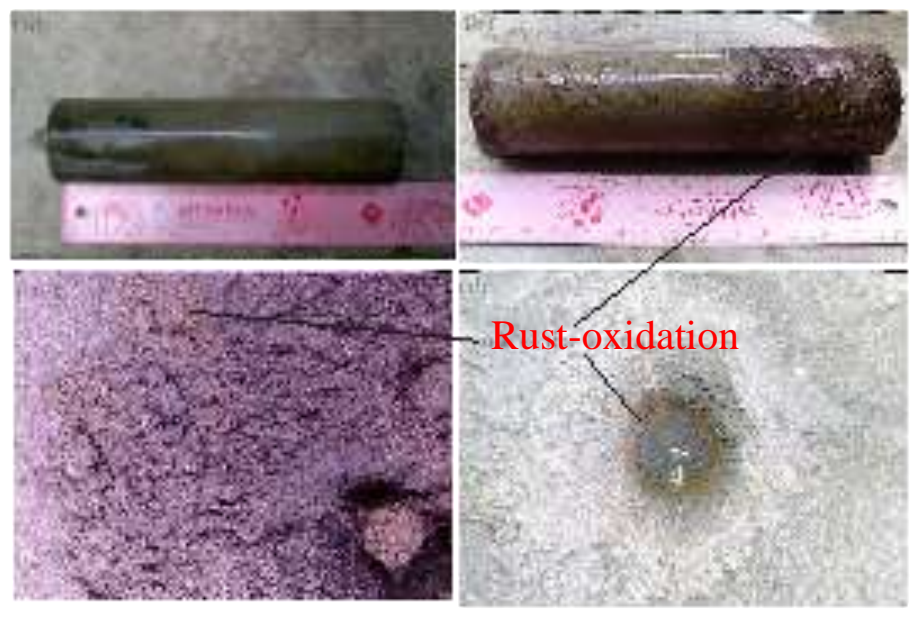

Figure 10. Metal cylinder target (a) Before observation (b) After observation, rust oxide in the bottom of the metals plate. 
Table 1: Measurement Schedules

\begin{tabular}{c|c|c|c|c}
\hline Point Name & Date & Start & Finish & Captions \\
\hline T-1 & Day 1 & $13: 47$ & $16: 58$ & Positions function \\
T-2 & Day 2 & $12: 27$ & $15: 40$ & Positions function \\
T-9 & Day 9 & $12: 14$ & $13: 33$ & Positions function \\
T-14 & Day 12 & $12: 07$ & $13: 21$ & Positions function \\
T-19 & Day 17 & $14: 14$ & $15: 24$ & Positions function \\
Monitoring & Day 23 & $08: 15$ & $22: 49$ & Time function \\
T-29 & Day 27 & $12: 16$ & $13: 18$ & No Target \\
\hline
\end{tabular}

\section{ACKNOWLEDGEMENT}

The authors for this paper would like to thank to Arief Rahmadi and Alfian Romadhoni for helping in the making of the aquarium model and other persons that could not be mentioned in this project

\section{REFERENCES}

[1] Campbell, D. L. 1977. Model for Estimating Electric Macro Anisotropy Coefficient of Aquifers with Horizontal and Vertical Fractures. Geophysics Journal, Vol. 42, Issue 1, pp.114-117. https://doi.org/10.1190/1.1440705

[2] Castermant, J., Mendonca, C.A., Revil, A., Trolard, F., and Bourrie, G., 2008, Redox potential distribution inferred from self-potential measurements associated with the corrosion of a burden metallic body, European Association of Geoscientists \& Engineers, Prancis

[3] Ekine, A.S., and Emujakporue, G. O. 2010, Investigation of Corrosion of Buried Oil Pipeline by the Electrical Geophysical Methods, Journal of Application Science and Enviroment, Nigeria vol. 14 (1), pp. 63-65

[4] Ernstson, K. and Scherer, H. U., 1986, Self-potential variations with time and their relation to hydrogeologic and meteorological parameters, Society of Exploration Geophysicist, Jerman, 51(10), 1967-1977. https://doi.org/10.1190/1.1442052

[5] Jee, K. H., Cheong, H. M., Kyung, J. W., Won, J. S., Park, J., and Lee, C. O., 2007, A Construction Case of Corrosion Monitoring of Marine Concrete Structures for Chloride Attack, International Construction Database, Korea Selatan

[6] Lobo-Guerrero, A., 2004, Application of spontaneous potential profiles for exploration of Goldrich epithermal low sulphidation veins in a humid region, Geological Society of South Africa, Johannesburg

[7] Lower, S. K., 2004, Electrochemistry: Chemical reactions at an electrode, galvanic and electrolytic cells, Kanada

[8] Nyquist J. E., Corry, C. E., 2002, Self-potential: The ugly duckling of environmental geophysics, Society of Exploration Geophysicist, US

[9] Oldham, W. H., 1959, Geophysical Survey of Nymagee Copper Field, Departement of National Development Bureau of Mineral Resource, Geology, and Geophysics, Selandia Baru

[10] Revil, A. and Jarandi, A., 2013, The Self-Potential Method: Theory and Application in Environmental Geosciences, Cambridge University Press, Cambridge

[11] Rittgers, J. B., Revil, A., Karaoulis, M., Mooney, M. A., Slater, L. D., and Atekwana, E. A., 2013, Self-potential signals generated by the corrosion of buried metallic objects with application to contaminant plumes, Society of Exploration Geophysicist, Colorado

[12] Roth, M. J. S., Mackey, J. R., Mackey, C., and Nyquist, J. E. 2002. A Case Study of the Reliability of Multielectrode Earth Resistivity Testing for Geotechnical Investigations in Karst Terrains. Engineering Geology, Vol. 65 Issues 2-3, pp. 225-232. https://doi.org/10.1016/S0013-7952(01)00132-6

[13] Sato, M., and Mooney, H. M., 1960, The Electrochemical and Mechanism of Sulfide Self-potentials, Society of Exploration Geophysicist, Cambridge.

[14] Ward, S. H., and Sill, W. R., 1978, Resistivity, Induced Polarization, and Self-Potential Methods in Geothermal Exploration, Earth Science Laboratory and Departement of Geology and Geophysics University of Utah, Utah. 